\title{
A successful launch
}

One year ago, the American Society for Clinical Investigation (ASCI) launched JCI Insight. I am pleased to report that the last year has been a resounding success for the Society's newest journal. Let me begin with some background. In my inaugural editorial, I laid out the rationale for JCI Insight (1). In brief, its mission is two-fold: to provide an additional forum for ASCI members and other authors to publish their work in a JCI-brand journal, and to provide financial sustainability to the Society, which has been strained by our model of complete free access to research papers. Over the past year, we have accepted nearly 300 high-quality papers for publication, many of which have gone on to be highly viewed, downloaded, and noticed by our peer journals for their important scientific discoveries. Moreover, the Society's finances have been remarkably stabilized, and the additional revenue made possible by the launch of JCI Insight has allowed us to eliminate submission fees for the Journal of Clinical Investigation (JCI), a longstanding concern of many authors. To say that all the hard work that the JCI Insight Editorial Board has spent over the past year poring over manuscripts has been gratifying would simply be an understatement! I am deeply grateful to the Editorial Board, consulting editors, and reviewers for their hard work and dedication in adjudicating manuscripts and providing fair and thoughtful peer review to authors.

One of the thoughts we had for JCI Insight was to use this journal as an incubator for innovative ways to publish data and provide peer review. Two examples of the ideas we implemented are the following: (i) in order to enhance data integrity and transparency, we ask authors to show their raw data in figures and to provide clear and informative figure legends, and (ii) we provide ASCI members guaranteed external peer review for their manuscripts and encourage members to help us identify the best reviewers who can judge their work. Indeed, the editorial philosophy at JCI Insight is to have reviewers provide comments on how their work can be improved, not list reasons for it to be rejected.

Given the well-known subjective nature and vagaries of peer review, I have been on a mission to minimize the current trend in peer review of demanding endless experimentation before a paper can be accepted for publication. I believe this slows down science and hurts the process of scientific discovery. To begin to counter this pernicious trend, we recently launched a new endeavor at JCI Insight, where we will accept reviews from another high-tier journal and will make an editorial decision based on those external peer reviews, most often without sending out the paper for re-review (see http://insight.jci.org/kiosks/ authors\#Editorial_Policies). It's too early to tell how this will work out, but so far, we have received terrific feedback from authors.

For JCI Insight, 2017 looks to be bright, but it will also be a year for transition. A new Editor in Chief for the $J C I$ is currently being deliberated upon and will begin in July, replacing the editorial board at Duke and UNC. Over the next year, and perhaps into 2018, your representatives on the ASCI Council, along with the new JCI Editor and I, will work to codify the Editorial Board structure and the business model for JCI Insight within the Society's bylaws. As an independent journal with a bright future, much thought will need to be given to how JCI Insight should evolve to best serve the needs of the Society. As was always planned, my role as Founding Editor in Chief at JCI Insight will end, and in the next 1 to 2 years, a new editor will be selected to take the helm of the Society's new shining star.

On behalf of the Editorial Board at JCI Insight, we are proud to serve the ASCI community and to continue our Society's founders' compelling legacy: to disseminate knowledge. It is a continuing honor and privilege to be Editor of JCI Insight, and as always, I welcome your thoughts and comments.

\section{Howard A. Rockman}

Editor in Chief, JCI Insight

\footnotetext{
1. Rockman HA. New kid on the block. J Clin Invest. 2016;126(1):1-2.
}

Published: January 26, 2017

Reference information: JCI Insight. 2017;2(2):e92800. doi:10.1172/jici.insight.92800. 